\title{
Seismic source displacement by coda wave interferometry at Soufrière Hills Volcano, Montserrat, WI
}

\author{
S. De Angelis \\ Alaska Earthquake Information Center, 903 Koyukuk Drive, Fairbanks, Alaska, 99775-7320, USA \\ Received: 16 April 2009 - Revised: 12 June 2009 - Accepted: 23 June 2009 - Published: 3 August 2009
}

\begin{abstract}
Since the start of the ongoing eruption, on 18 July 1995, the Soufrière Hills Volcano (SHV) on Montserrat, WI, has been monitored with a network of seismometers providing an unparalleled wealth of observations. A recurrent and intriguing feature of the seismicity at SHV is the occurrence of regular swarms of low-frequency earthquakes during episodes of volcanic unrest. A notable example of this type of activity was recorded during the summer of 2008 when SHV erupted, on 29 July, following 15 days of seismic unrest. An impressive swarm of low-frequency earthquakes with strikingly similar waveforms, was recorded on 26 July through 27 July 2008. In this paper, the cross-correlation properties of the repeating earthquakes are investigated, and coda wave interferometry methods applied to infer seismic source displacement throughout the swarm.
\end{abstract}

\section{Introduction}

The Soufrière Hills Volcano (SHV) on Montserrat, West Indies, is an andesitic dome building volcano situated near the Northern end of the Lesser Antilles volcanic arc. Seismic activity at SHV is monitored, by the Montserrat Volcano Observatory (MVO), with a network of 9 broadband ( $\mathrm{T}=30 \mathrm{~s}$ ), three-component Guralp CMG-40T seismometers, and 2 short period ( $\mathrm{T}=1 \mathrm{~s})$ Mark Products L4 vertical instruments (Fig. 1).

The ongoing eruption of SHV began on 18 July 1995 (Aspinall et al., 1998), and it has encompassed three main cycles of dome growth and collapse: September 1995 to March 1998; November 1999 to July 2003; August 2005 to present. High extrusion rates between August 2006 and January 2007

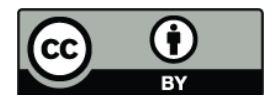

Correspondence to: S. De Angelis (silvio@giseis.alaska.edu) have contributed to build the present summit lava dome with an estimated volume of about $200 \cdot 10^{6} \mathrm{~m}^{3}$. In January 2007 , a minor explosive event removed a small portion of the dome (De Angelis et al., 2007), and marked the beginning of a 15-month period of quiescence in seismic and surface activity. This pause ended during May 2008 when increasing earthquake activity was recorded at SHV leading to a sequence of pyroclastic explosions that culminated in a shortlived, but powerful, vulcanian eruption on 29 July 2008. Recent eruptions of SHV have been accompanied by swarms of self-similar, long-period $(1-5 \mathrm{~Hz})$ and hybrid $(1-10 \mathrm{~Hz})$ earthquakes, a type of activity also reported at other active lava dome building volcanoes worldwide (Moran et al., 2007). There have been several periods between 1996 and 1998 when frequent swarms of low-frequency earthquakes (LF, long-period and hybrids collectively), at SHV, have preceded episodes of partial dome collapse or vulcanian explosions (Miller et al., 1998). These swarms often begun with earthquakes a few minutes apart (either regularly or irregularly spaced), becoming closer as time progressed, and eventually merging into a quasi-continuous signal where discrete events could still be recognized through the presence of gliding spectral lines (Powell and Neuberg, 2003). The duration of individual swarms could range from few to several hours, and they were recorded in episodes lasting days to months. Within swarms, waveforms could be nearly identical, consistent with the hypothesis of relatively small source volumes (Ottemoller, 2008). Green and Neuberg (2006) recognized the existence of groups, or families, of similar LF earthquakes, and suggested a relation between the relative timing of these families and cyclic tilt observed at SHV.

Despite the work of a number of researchers aiming to understand LF volcanic seismicity, the interpretation of its source mechanisms and propagation effects is still controversial. Waveforms recorded at volcanoes are often noisy and contain peculiar spectral components arising from complex

Published by Copernicus Publications on behalf of the European Geosciences Union. 


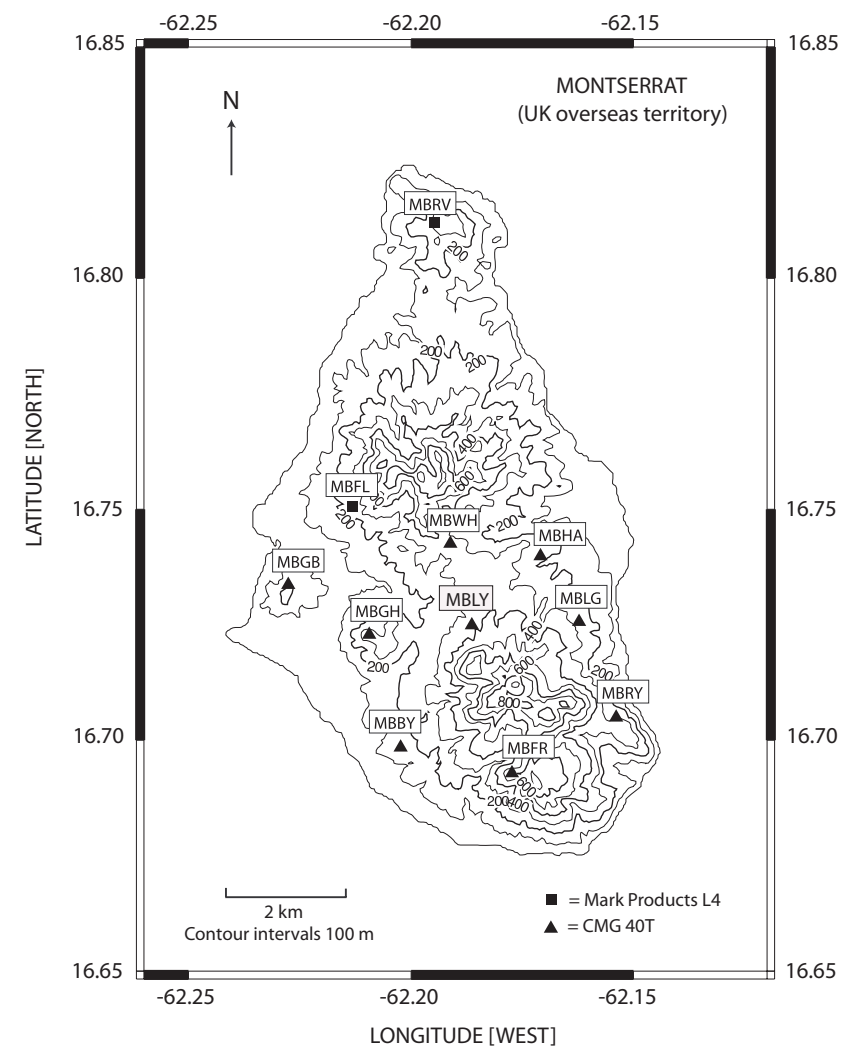

Fig. 1. Seismic network at Soufrière Hills Volcano on Montserrat, West Indies, operated by the Montserrat Volcano Observatory (MVO).

interactions of tectonic and magmatic processes. Besides, volcano monitoring networks often consist of a relatively small number of stations with marginal coverage, providing a challenge for seismic investigation in such complex and highly heterogeneous settings. Typical long-period events, at the lower end of the LF spectrum $(1-4 \mathrm{~Hz})$, have been often ascribed to volumetric sources involving motion of fluids within resonating volcanic conduits and dikes (Chouet, 1996). Hybrids, often considered members of the LF family of earthquakes (Green and Neuberg, 2006), are characterized by high-frequency and impulsive onsets followed by a long-period ringing coda. Tuffen at al. (2005) and Neuberg et al. (2006), suggested that the high-frequency onset reflects the seismic source, represented as fracturing and faulting within the erupting magma. Magma fracturing, in turn, would trigger conduit resonance, thus, generating the lower frequencies observed in the coda of hybrid earthquakes. Iverson et al. (2006) proposed a physical model to explain the origin of repeating LF earthquakes at Mt. St. Helens as the result of stick-slip motion along the margins of a viscous lava plug forced upwards by ascending magma. Harrington and Brodsky (2006), argued that hybrid earthquakes recorded at different volcanoes exhibit corner frequencies and seismic magnitude scaling consistent with brittle-failure sources.
They suggested that the LF part of the hybrid spectrum may be explained as the combined effect of complex propagation paths and low rupture velocities along the fault.

In contrast to the lively scientific debate on the source mechanisms of LF volcanic seismicity, there is almost unanimous consensus that repeating waveforms reflect stable sources confined within a small volume. Although, in some instances, the degree of repeatability of the recorded earthquakes is spectacular, as time progresses waveforms exhibit small variations, and subtle differences can be identified in the coda of similar events. In a strongly heterogeneous environment, such as a volcano, seismic waves that travel from the source to a receiver are scattered multiple times; the coda of seismograms, composed of these scattered waves, samples the medium more effectively than ballistic arrivals. Exploiting the cross-correlation properties of the repeating waveforms, by use of coda wave interferometry (CWI), it is possible to separate temporal changes of the material and source properties, from the spatial variation of the earthquake source.

In this paper a swarm of repeating LF earthquakes, recorded at SHV during 26-27 July 2008, is investigated. CWI is applied to detect and characterize subtle changes in the waveforms throughout the duration of the swarm.

\section{Activity at SHV during July 2008}

Starting on 21 July through 29 July 2008 the SHV seismic network recorded elevated levels of seismicity. Irregular swarms of VT earthquakes characterized the period between 21 July and 26 July. Seismicity started to increase remarkably at approximately 04:00 a.m. (all times in the manuscript are Coordinated Universal Time, UTC, unless otherwise stated), on 26 July, marking the onset of regular earthquake activity with LF character. The number and size of the events increased, peaking at about 01:00 a.m., on 27 July. At approximately 05:00 a.m., on 27 July, seismicity evolved from discrete LF earthquakes to short (1-2 min duration) bursts of LF tremor. A series of small surface explosions were then observed. The first and largest of such events occurred at approximately 01:35 p.m., it vented ash for about $15 \mathrm{~min}$ generating a plume that reached a height of about $2.5 \mathrm{~km}$ above sea level (a.s.l.). Two other eruptive events were observed in the following $45 \mathrm{~min}$. Seismic activity continued at a reduced level until 02:00 p.m. on 28 July. By 07:30 p.m. seismicity had returned nearly to background levels. Few hours later, at 03:27 a.m. on 29 July, a vulcanian eruption occurred without further warning. The eruption generated a large ash column that reached a height of $12 \mathrm{~km}$ a.s.l, pyroclastic flows with run-out distances of approximately $1.5 \mathrm{~km}$, and extensive fallout of airborne pumice. 

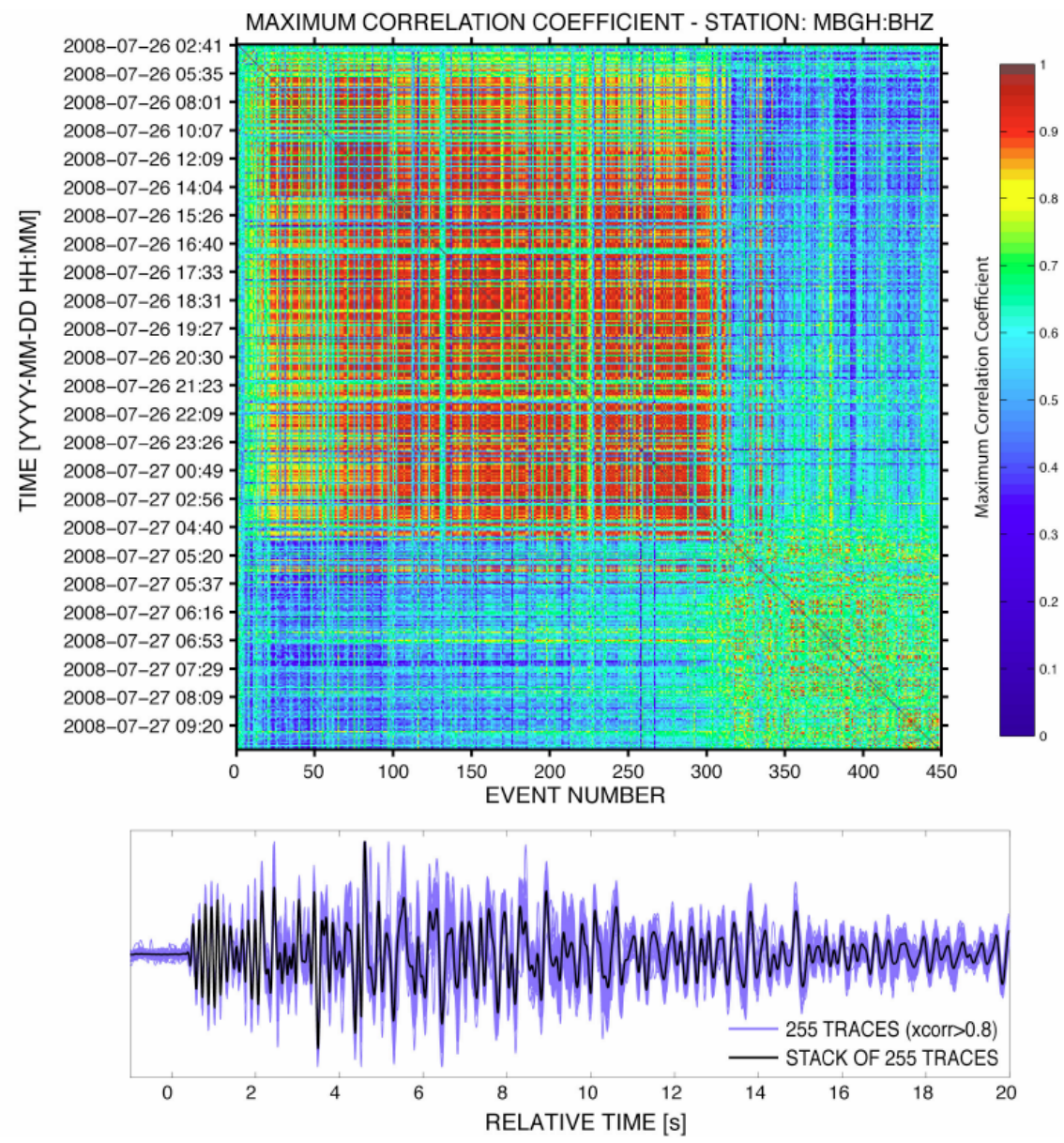

Fig. 2. (Top) Cross-correlation matrix showing an obvious cluster of repeating earthquakes recorded at seismograph station MBGH (vertical component) on 26-27 July 2008; (Bottom) Plot of 255 traces with correlation coefficient $>0.8$ extracted from the cross-correlation matrix and their average.

\section{The repeating low-frequency earthquakes}

A total of 1588 earthquakes were recorded during the three days preceding the eruption, 277 large enough to be located underneath the dome at depths of 0 to $3.4 \mathrm{~km}$ below sea level. A cross-correlation algorithm, applied to the seismic records, revealed striking waveform similarity during this period. The cross-correlation matrix in Fig. 2 shows an obvious cluster of repeating waveforms as recorded on the vertical component at station MBGH during 26-27 July. The horizontal component seismograms exhibit a similar pattern although the lower signal-to-noise ratio results in a lesser degree of correlation. Noticeably, high waveform similarity was consistently observed across the network (Fig. 3) suggesting a stable source mechanism rather than site-specific effects. Constraints on the properties of the repeating earthquakes have been obtained by use of CWI. A procedure proposed by Snieder et al. (2006) was applied to data from station MBGH in order to identify small variations in the waveforms. Hourlong stacks were extracted from the earthquake records be- tween 26 July, 04:00 a.m and 27 July, 05:00 a.m. The stacked waveforms are showed in Fig. 4; each of these traces represents an average of 5 to 15 events. In the manuscript, the difference in time between the stacked traces will be referred to as age in contrast with time measured along the seismic records.

Preliminary inspection of the stacked signals revealed variations in the coda of seismograms from age 0 (bottom trace in Fig. 4) through the following $24 \mathrm{~h}$. The time shift between the trace at age 0 and waveforms for later ages was evaluated by peaking the maximum of the time-shifted crosscorrelation (2-s moving window). No evidence of systematic phase shifts was found, suggesting that the loss of correlation in the coda should not be attributed to variations of the seismic velocity in the volcanic medium (Snieder and Hagerty, 2004). Alternative explanations for the decreasing degree of waveform correlation, may include variations of the sourcetime function or source displacement. Changes in the sourcetime function should have an effect on early wavelets, at least, comparable to coda variations. In order to test this 

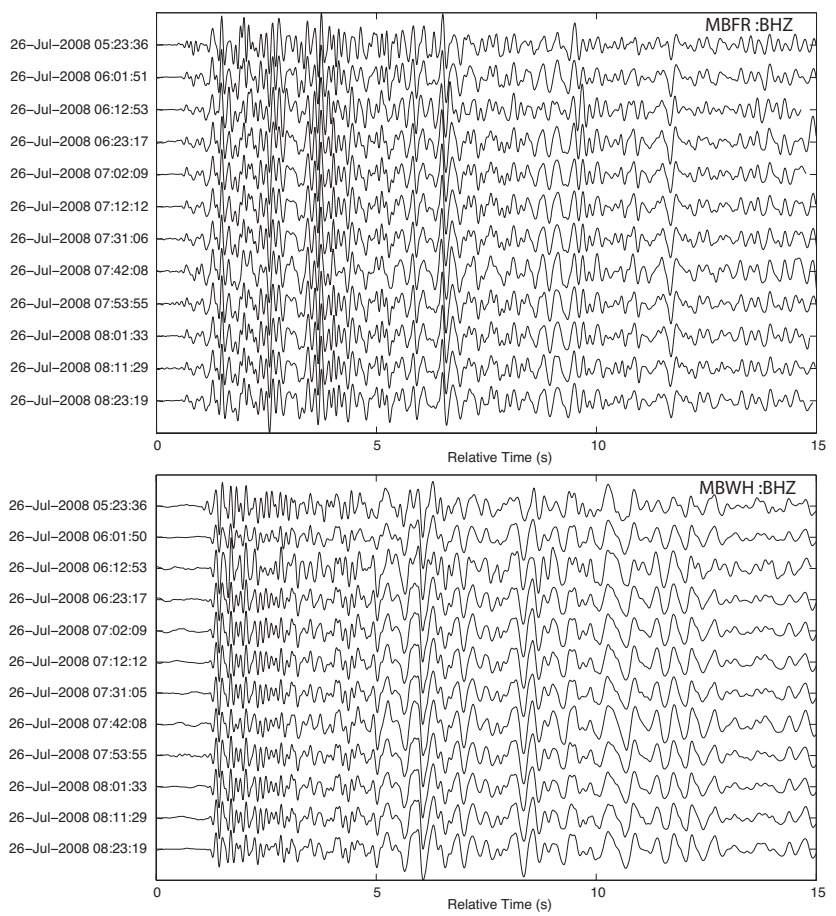

Fig. 3. Sample LF repeating waveforms recorded at seismic stations MBFR and MBWH (vertical component) showing a high degree of waveform similarity across the SHV network.

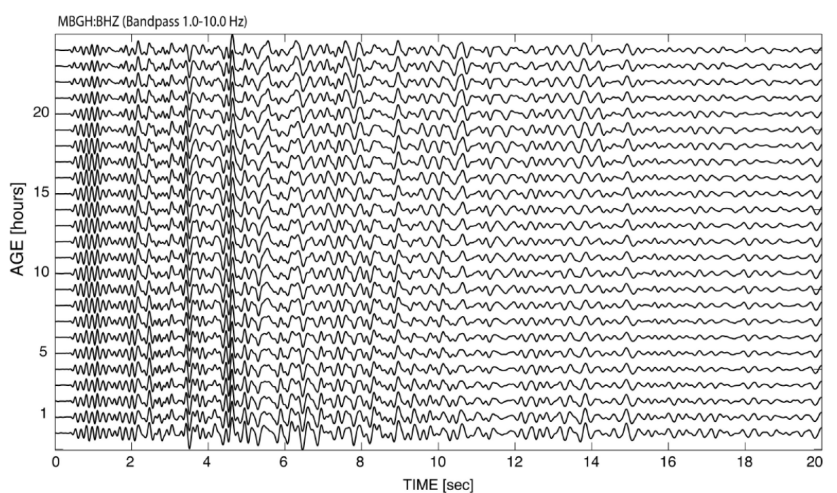

Fig. 4. Hour-long stacked seismograms extracted from earthquake records between 26 July, 04:00 a.m. (UTC) and 27 July, 05:00 a.m. (UTC). The difference in time between stacked traces (vertical axis) is referred to as age; horizontal axis is time, measured in seconds along the seismic records.

hypothesis, the normalized cross-correlation coefficient was calculated for the early $(0-5 \mathrm{~s})$ and late $(5-10 \mathrm{~s})$ part of the seismograms of Fig. 4, using the signal at age 0 as a reference. The results, presented in Fig. 5, demonstrate that whilst correlation for the early seismograms is consistently high throughout the duration of the swarm, the degree of similarity of the signal decreases with time for the late part of the waveforms. Similar observations from other volcanoes have been reported in literature (e.g. Gret et al., 2005). The early seismogram is highly reproducible, leading to discard the hypothesis of significant variations of the source-time function. Small variations of the source position were, thus, considered the most likely explanation for the changes in the seismograms. Although this cannot be conclusively proved, it is possible to evaluate the amount of source displacement required to produce the observed variations.

\section{The source displacement}

Seismic source separation for repeating earthquakes can be estimated using CWI methods. When a seismic source is displaced, its distance to scatterers in the surrounding medium changes. The result is a perturbation of the arrival times of the waves that interfere to produce the seismic coda. The degree of correlation of the coda waves for the original and displaced source is a function of the variance of the traveltime perturbation. In particular, the correlation coefficient, $R$, is related to the variance of the travel-time perturbation, $\sigma_{\tau}$, and to the frequency, $\bar{\omega}^{2}$, according to the following relationship (Snieder, 2003):

$R=1-\frac{1}{2} \bar{\omega}^{2} \sigma_{\tau}^{2}$

The frequency, $\bar{\omega}^{2}$, can be calculated from the seismogram data, $u(t)$ :

$\bar{\omega}^{2}=\frac{\int_{t-T}^{t+T} \dot{u}^{2}\left(t^{\prime}\right) d t^{\prime}}{\int_{t-T}^{t+T} u^{2}\left(t^{\prime}\right) d t^{\prime}}$

where the integral is performed over a window of length $2 T$ centred at time $t$. The relationship between the variance of the travel-time perturbation and the inferred source displacement depends on the source mechanism. Snieder and Vrijlandt (2005), have demonstrated this relationship for different types of source. If displacement occurs, for instance, along the fault plane, the source dislocation, $\delta$, is given by:

$\delta=\left[7\left(\frac{2}{v_{p}^{6}}+\frac{3}{v_{s}^{6}}\right) /\left(\frac{6}{v_{p}^{8}}+\frac{7}{v_{s}^{8}}\right)\right]^{\frac{1}{2}} \sigma_{\tau}$

where $v_{p}$ and $v_{s}$ are $P$ - and $S$-wave velocity in the medium. The displacement for a source moving in a direction perpendicular to the fault plane and one with an isotropic explosive mechanisms, is expected to be 1.33 and 1.72 times larger than Eq. (3), respectively.

Equation (3) was applied to the SHV data in order to evaluate the source displacement associated with the changes in the coda of seismograms. The correlation coefficient between all stacked traces of Fig. 4 and the signal at age 0 was calculated in different frequency bands $(1-2 \mathrm{~Hz}, 2-5 \mathrm{~Hz}$, and $5-10 \mathrm{~Hz})$ and time windows $(1-5 \mathrm{~s}, 5-10 \mathrm{~s}, 10-15 \mathrm{~s}, 15-$ $20 \mathrm{~s}$ ) along the seismograms, and converted to source displacement (Fig. 6). The calculations were based on a $P$-wave 

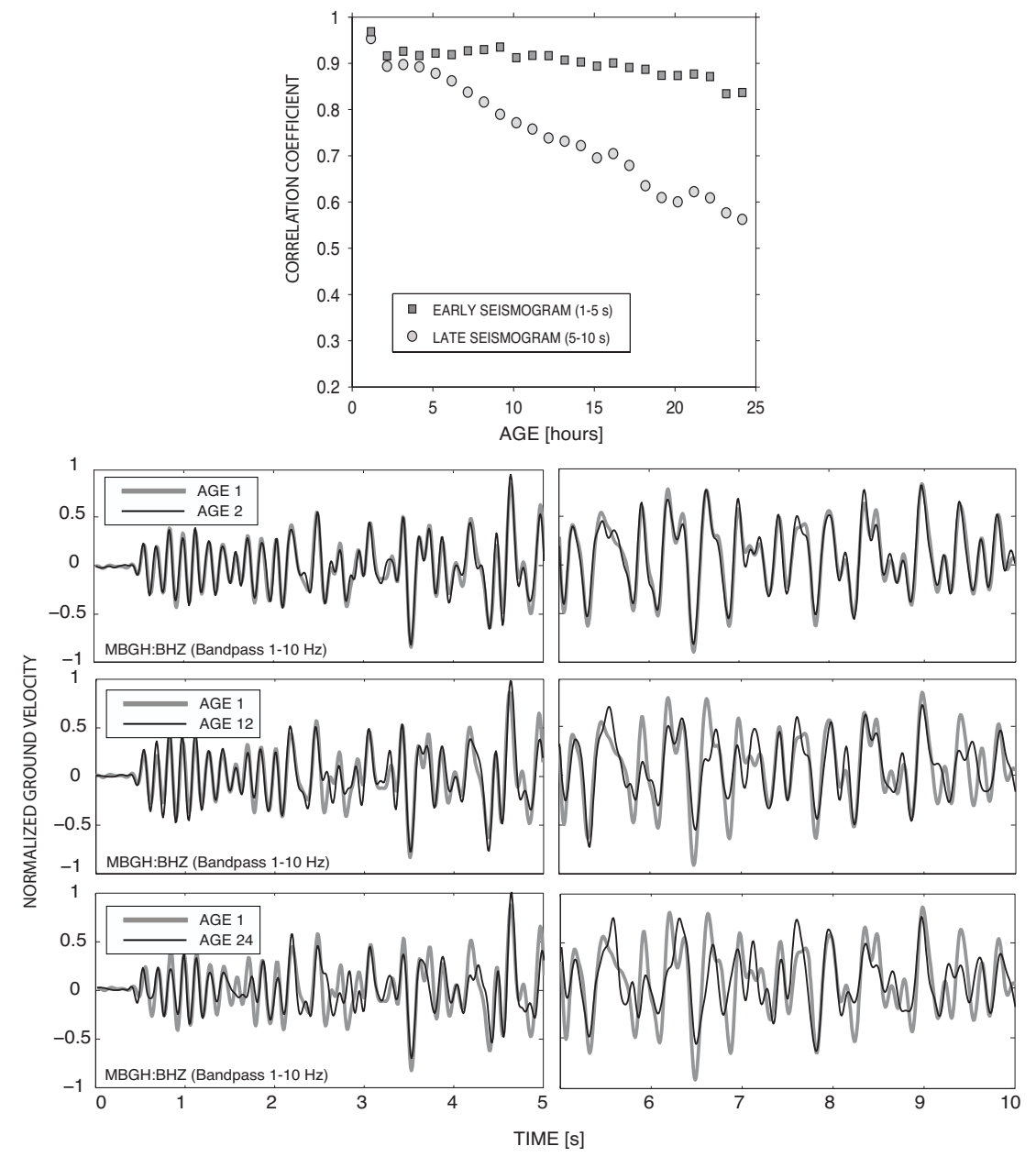

Fig. 5. (Top) Variations of the correlation coefficient for early (1-5s) and late (5-10s) seismograms of Fig. 4. Events for later ages are correlated with age 0 (bottom trace of Fig. 4); (Bottom) Superposition of stacked waveforms corresponding to age 2, 12, 24 (thin black line) with age 1 (thick grey line), for early (1-5s) and late (5-10 s) seismograms.

velocity of $3.2 \mathrm{~km} / \mathrm{s}$ and $P$-wave to $S$-wave velocity ratio of 1.75 , consistent with the velocity model used for earthquake locations at SHV. Equation (3) is sensitive to the seismic velocity model employed, and efforts should be undertaken in the direction of improving its definition at SHV. However, uncertainties related to the velocity model employed, do not appear to affect the results dramatically, and they represent a second-order effect in comparison to the scarce knowledge of the source mechanism. A cumulative source displacement of about $235 \mathrm{~m}$ (Fig. 7) was obtained averaging the data of Fig. 6. Error bars in Fig. 7 represent the standard deviation of displacement data and do not take into account errors due to the velocity model employed. The slope of the curve in Fig. 7, suggests a rather steady rate of source displacement.

It must be noted that the CWI method relies on a number of simplifying assumptions: the velocity field is considered isotropic and the medium locally homogenous, mode conversion at the free surface is ignored, and the source separation must be less than a wavelength. However, results from its application have been validated by comparison with other techniques such as the double-difference relocation method (Waldhauser and Ellsworth, 2000; Snieder and Vrijlandt, 2005). Whilst most methods for relative earthquake location require seismograms from multiple stations, CWI has the undoubted advantage to provide an estimate of source separation only employing waveforms recorded at a single site. This is particularly attractive for volcano monitoring operations as it makes the rapid estimate of the evolution of earthquake swarms feasible. Furthermore, methods that only require records from a small number of stations are especially desirable as seismic networks for volcano monitoring are often sparse, and the quality of data is heavily affected by the harsh environment.

A swarm of repeating LF earthquakes culminating in an eruption strongly suggests that this represents the seismic manifestation of rising magma. This type of seismicity regularly accompanies the ascent of viscous magma at volcanoes and it is has been associated, in some instances, with lava 

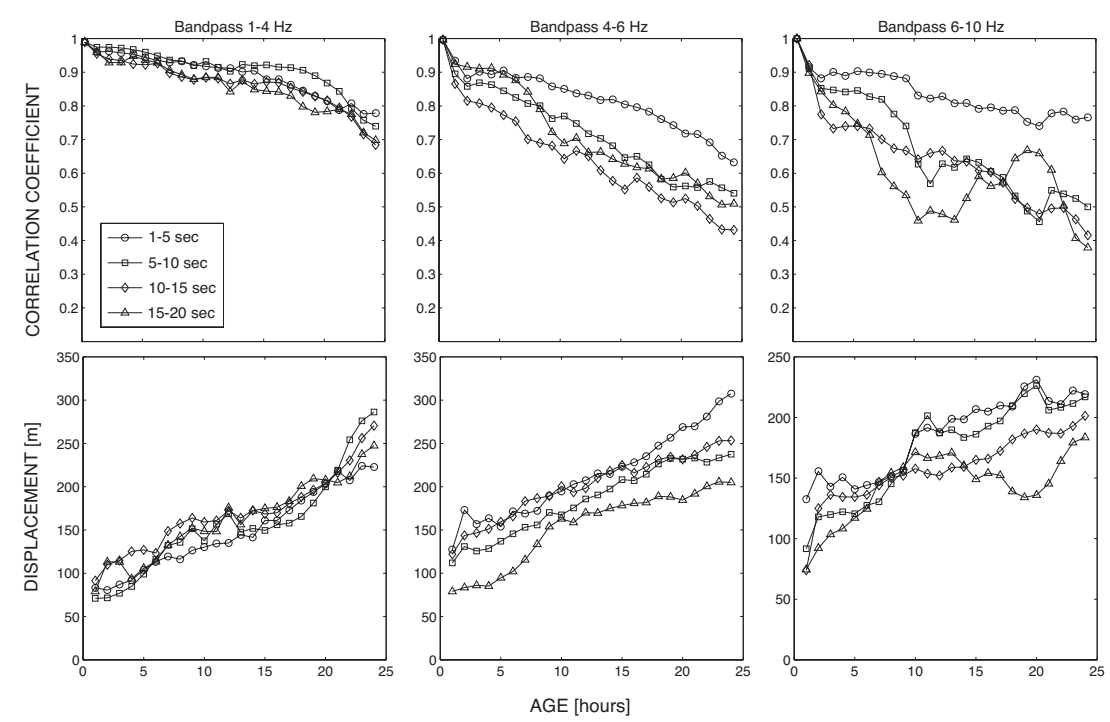

Fig. 6. (Top) Correlation coefficient between all waveforms and the bottom trace of Fig. 4, for data band-passed between 1-4 Hz (left), 4$6 \mathrm{~Hz}$ (middle), and 6-10 Hz (right). Correlation coefficients are computed for the time windows $0-5 \mathrm{~s}, 5-10 \mathrm{~s}, 10-15 \mathrm{~s}$, and 15-20 s; (Bottom) Source displacements from age 0 (bottom trace in Fig. 4) through later ages calculated according to Eq. (3) (see text).

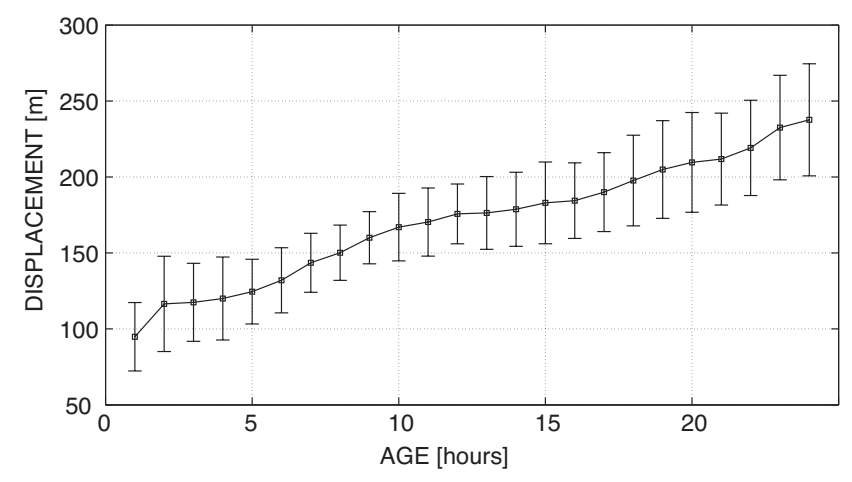

Fig. 7. Cumulative seismic source displacement and its variance as obtained averaging the data presented in Fig. 6

extrusion and dome growth (Iverson et al., 2006). There is no obvious evidence that magma reached the surface (lava dome extrusion) at SHV during the phase of repeating seismicity during 26-27 July. However, viscous magma could have been extruded from a stalled crustal reservoir, into an overlying conduit system during this time, possibly encouraged by the injection of fresh, more mafic, melt from depth. This may have triggered the ascent of a slug at the top of the magma column that caused earthquake activity. The calculated source displacement suggests that magma ascent may have been fast enough to prevent efficient degassing, thus, building an explosive potential for the eruption. When the slug reached the surface, fragmentation was triggered and propagated downward into the magma column producing a powerful explosive event. The relatively short duration of the event may be attributed to the fact that the influx of magma from depth into the shallow reservoir/conduit system was not able to keep pace with the rapid drainage of magma, hence, sutstaining the eruption. Whilst ascent of viscous magma within the volcanic conduit is a reasonable source for repeating seismicity, the exact mechanisms that produce these regular waveforms at SHV, are not yet known. This type of earthquakes still remains object of speculation and debate as well as one of the most interesting research targets in volcanic seismology.

\section{Conclusions}

CWI was applied to a pre-eruption swarm of repeating LF earthquakes, recorded during July 2008 at SHV; the objective was to separate temporal changes of the material and source properties, from spatial variations of the earthquake source. Small changes detected in the coda of similar earthquakes were quantified by waveform cross-correlation. The lack of systematic time shifts in the waveforms, argues against changes in the properties of the volcanic material to produce these variations. The high similarity of the early part of the seismograms suggests that the source-time function didn't change significantly throughout the swarm. The measured waveform variations appear to reflect movement of the seismic source with time. A source displacement of $235 \mathrm{~m}$ over the span of a day was calculated, using seismic interferometry. Seismic and observational evidence suggests that the source of repeating seismicity at SHV may be linked to preeruption ascent of magma.

Whilst it remains inherently difficult to decipher the nature of the seismic source, the observations and the results of the analyses presented in this paper may contribute to constrain 
its mechanisms, spatial and temporal evolution. Development of methods and algorithms to detect, classify, and quantify volcanic seismicity in a timely fashion (Beyreuther et al., 2008 ) is of crucial importance to volcano monitoring operations. Special attention should be dedicated to the study of LF earthquakes, as they constitute a characteristic type of precursory activity at active volcanoes. Further investigation, possibly involving deployment of dense networks of seismometers at SHV is highly desirable, as it would allow to constraint seismic source mechanisms, thoroughly investigate path effects, and characterize temporal variations of the local stress field around the volcano during periods of unrest.

Acknowledgements. The author is grateful to C. "Pyiko" Williams, R. "Tappy" Syers, M. Fergus, and V. Bass, of the Montserrat Volcano Observatory for their continuing efforts to maintain the Soufrière Hills Volcano seismic network operational. The author thanks S. De la Cruz-Reyna and R. Carniel for insightful comments that helped to improve the manuscript.

Edited by: J. Marti

Reviewed by: R. Carniel and S. De la Cruz-Reyna

\section{References}

Aspinall, W. P., Miller, A. D., Lynch, L. L., Latchman, J. L., Stewart, R. C., White, R. A., and Power, J. P.: Soufrière Hills eruption, Montserrat, 1995-1997: Volcanic earthquake locations and fault plane solutions, Geophys. Res. Lett. 25, 3397-3400, 1998.

Beyreuther, M., Carniel, R., and Wassermann, J.: Continuous Hidden Markov Models: Application to automatic earthquake detection and classification at Las Canãdas caldera, Tenerife, J. Volcanol. Geoth. Res., 176(4), 513-518, doi:10.1016/j.jvolgeores.2008.04.021, 2008.

Chouet, B. A.: Long-period volcano seismicity: its source and use in eruption forecasting, Nature, 380, 309-316, 1996.

De Angelis, S., Bass, V., Hards, V., and Ryan, G.: Seismic characterization of pyroclastic flow activity at Soufrire Hills Volcano, Montserrat, 8 January 2007, Nat. Hazards Earth Syst. Sci., 7, 467-472, 2007,

http://www.nat-hazards-earth-syst-sci.net/7/467/2007/.

Green, D. and Neuberg, J.: Waveform classification of volcanic low-frequency swarms and its implication, J. Volcanol. Geoth. Res., doi:10.1016/j.jvolgeores.2005.08.008, 2006

Harrington, R.M., Brodsky, E.E. : Volcanic hybrid earthquakes that are brittle-failure events, Geophys. Res. Lett. 34, L06308, DOI:10.1029/2006GL02871, 2006

Gret, A., Snieder, R., Aster, R.C., Kyle P.R.: Monitoring rapid temporal change in a volcano with coda wave interferometry, Geophys. Res. Lett., 32, L06304, doi:10.1029/2004GL021143, 2005

Iverson, R. M., Dzurisin, D., Gardner, C. A., Gerlach, T. M., LaHusen, R. G., Lisowski, M., Major, J. J., Malone, S. D., Messerich, J. A., Moran, S. C., Pallister, J. S., Qamar, A., Schiling, S. P., and Vallance, J. W.: Dynamics of seismogenic volcanic extrusion at Mount St. Helens in 2004-2005, Nature, 444, 439-443, 2006.
Miller, A. D., Stewart, R. C., White, R. A., Luckett, R., Baptie, B. J., Aspinall, W. P., Latchman, J. L., Lynch, L. L., and Voight, B.: Seismicity associated with growth and collapse at the Soufrière Hills Volcano, Montserrat, Geophys. Res. Lett., 25, 3401-3404, 1998.

Moran, S. C., Malone, S. D., Qamar A. I., Thelen, W., Wright, A. K., and Caplan-Auerbach, J.: Seismicity Associated with Renewed Dome-Building at Mount St. Helens, 2004-2005, in: A Volcano Rekindled: The Renewed Eruption of Mount St. Helens, edited by: Sherrod, D. R., Scott, W. E., and Stauffer, P. H., 2004-2006, US Geological Survey Professional Paper 1750, 2007

Neuberg, J., Tuffen, H., Collier, L., Green, D., Powell, T.: The trigger mechanism of low-frequency earthquakes on Montserrat, J. Volcanol. Geoth. Res., 133(1-2), 37-50, doi:10.1016/j.jvolgeores.2005.08.008, 2006.

Ottemoller, L.: Seismic hybrid swarm precursory to a major lava dome collapse: 9-12 July 2003, Soufrière Hills Volcano, Montserrat, J. Volcanol. Geotherm. Res., 177(4), 903-910 , doi:10.1016/j.jvolgeores.2008.07.002, 2008.

Prejean, S., Snieder, R., Johnson, J.B.: Observational Evidence for a Rapidly Moving Seismic Source at Mount St. Helens, American Geophysical Union, Fall Meeting 2007, V51D-0781, 2007.

Powell, T. W. and Neuberg, J.: Time dependent features in tremor spectra, J. Volcanol. Geotherm. Res., 128, 1-3, doi:10.1016/S0377-0273(03)00253-1, 2003.

Snieder, R.: Constraining relative source locations with the seismic coda, Center for Wave Phenomena Consortium Project 2003 , CWP-458, 2003.

Snieder, R., Hagerty, M.: Monitoring change in volcanic interiors using coda wave interferometry: Application to Arenal Volcano, Costa Rica, Geophys. Res. Lett. 31, L09608, doi:10.1029/2004GL019670, 2004.

Snieder, R., Vrijlandt, M.: Constraining Relative Source Locations with Coda Wave Interferometry: Theory and Application to Earthquake Doublets in the Hayward Fault, California, J. Geophys. Res., 110, B04301, doi:10.1029/2004JB003317, 2005.

Snieder, R., Prejean, S., and Johnson, J. B.: Spatial variation in Mount St. Helens clones from coda wave analysis, Center for Wave Phenomena Consortium Project 2006, CWP-543, 2006.

Tuffen, H., Dingwell, D. B.: Fault textures in volcanic conduits: evidence for seismic trigger mechanisms during silicic eruptions, Bull. Volcanol., 67, 370-387, 2005.

Waldhauser, F. and Ellsworth, W. L.: a double-difference earthquake location algorithm: Method and application to the northern Hayward fault, California, B. Seismol. Soc. Am., 90, 13531368, 2000. 